\title{
Spectral variations of the X-ray binary pulsar LMC X-4 during its long period intensity variation and a comparison with Her X-1
}

\author{
S. Naik and B. Paul
}

\begin{abstract}
Tata Institute of Fundamental Research, Homi Bhabha Road, Mumbai - 400005, India
Received 3 May 2002 / Accepted 6 January 2003

Abstract. We present spectral variations of the binary X-ray pulsar LMC X-4 using the RXTE/PCA observations at different phases of its 30.5 day long super-orbital period. Only out of eclipse data were used for this study. During the high state of the super-orbital period of LMC X-4, the spectrum is well described by a high energy cut-off power-law with a photon index in the range of $0.7-1.0$ and an iron emission line. In the low state, the spectrum is found to be flatter with power-law photon index in the range $0.5-0.7$. A direct correlation is detected between the continuum flux in 7-25 keV energy band and the iron emission line flux. The equivalent width of the iron emission line is found to be highly variable during the low intensity state, whereas it remains almost constant during the high intensity state of the super-orbital period. It is observed that the spectral variations in LMC X-4 are similar to those of Her X-1 (using RXTE/PCA data). These results suggest that the geometry of the region where the iron line is produced and its visibility with respect to the phase of the super-orbital period is similar in LMC X-4 and Her X-1. A remarkable difference between these two systems is a highly variable absorption column density with phase of the super-orbital period that is observed in Her X-1 but not in LMC X-4.
\end{abstract}

Key words. stars: neutron - stars: pulsars: individual: LMC X-4, Her X-1 - X-rays: stars

\section{Introduction}

LMC X-4 is an eclipsing high-mass disk-fed accretionpowered binary X-ray pulsar in the Large Magellanic Cloud. It was first detected with the Uhuru satellite. The optical counterpart of the pulsar is found to be a 14th magnitude OB star (Sanduleak \& Philip 1977). Photometric and spectroscopic observations of the star revealed strong ellipsoidal light variations which indicated a binary period of 1.408 (Chevalier \& Ilovaisky 1977). X-ray pulsations with a spin period of $13.5 \mathrm{~s}$ were discovered by Kelley et al. (1983) which was later detected in the EXOSAT observations of the source even during the quiescent period and several 1 minute flares (Pietsch et al. 1985). X-ray eclipses with a 1.4 recurring period were discovered by Li et al. (1978) and White (1978). The X-ray intensity varies by a factor of $\sim 60$ between high and low states with a periodic cycle time of 30.5 day (Lang et al. 1981). The 30.5 day intensity variation has also been detected with the All Sky Monitor (ASM) detectors of GINGA and The Rossi X-ray Timing Explorer (RXTE) (Paul \& Kitamoto 2002). Flux modulation at super-orbital period in LMC X-4 is believed to be due to blockage of the direct X-ray beam by its precessing tilted accretion disk, as in the archetypal system Her X-1. Flaring events of duration ranging from $\sim 20 \mathrm{~s}$ to $45 \mathrm{~min}$ (Levine et al. 1991 and references therein) are seen about once in a day during which the source intensity increases by factors up to $\sim 20$.

GINGA and ROSAT observations showed that the X-ray spectrum of LMC X-4 in $0.2-30 \mathrm{keV}$ energy range can be

Send offprint requests to: S. Naik, e-mail: sachi@tifr.res.in modeled with a power law component with photon index $\alpha \sim$ 0.7 , a high energy cutoff $E_{\mathrm{c}} \sim 16 \mathrm{keV}$ and a folding energy $E_{\mathrm{F}} \sim 36 \mathrm{keV}$, a thermal bremsstrahlung component with temperature of $0.35 \mathrm{keV}$ and a blackbody with $k T \sim 0.03 \mathrm{keV}$ (Woo et al. 1996). The spectrum also shows a broad iron emission line at $E \sim 6.6 \mathrm{keV}$. The soft excess, whether modeled as a thermal Bremsstrahlung or a black-body type emission, shows modulation at the pulse period (Woo et al. 1996 and Paul et al. 2002). Paul et al. (2002) also argued that the Bremsstrahlung model of LMC X-4 is physically not acceptable due to its pulsating nature. The soft excess, also detected with BeppoSAX (La Barbera et al. 2001) was modeled as blackbody emission from the accretion disk at the magnetospheric radius Comptonized by moderately hot electrons in a corona above the accretion disk. La Barbera et al. (2001) also reported the presence of a cyclotron absorption line at $\sim 100 \mathrm{keV}$ in the $0.12-100 \mathrm{keV}$ energy spectrum of the source.

The accretion-powered X-ray pulsar Her X-1 with a spin period of $1.24 \mathrm{~s}$ and orbital period of 1.7 days is another binary system which possesses a long-term cycle of 35 days in $\mathrm{X}$-ray intensity, analogous to LMC X-4. The long-term intensity variation in Her X-1 is believed to be due to the obscuration by a precessing tilted accretion disk around the central neutron star counter rotating with respect to the orbital motion (Gerend $\&$ Boynton 1976). The X-ray light curve of the source over 35 day super-orbital period consists of main-on X-ray state with a mean duration of $\sim 7$ orbital periods which is surrounded by two off-states each of $\sim 4$ orbital cycles and a short-on state 


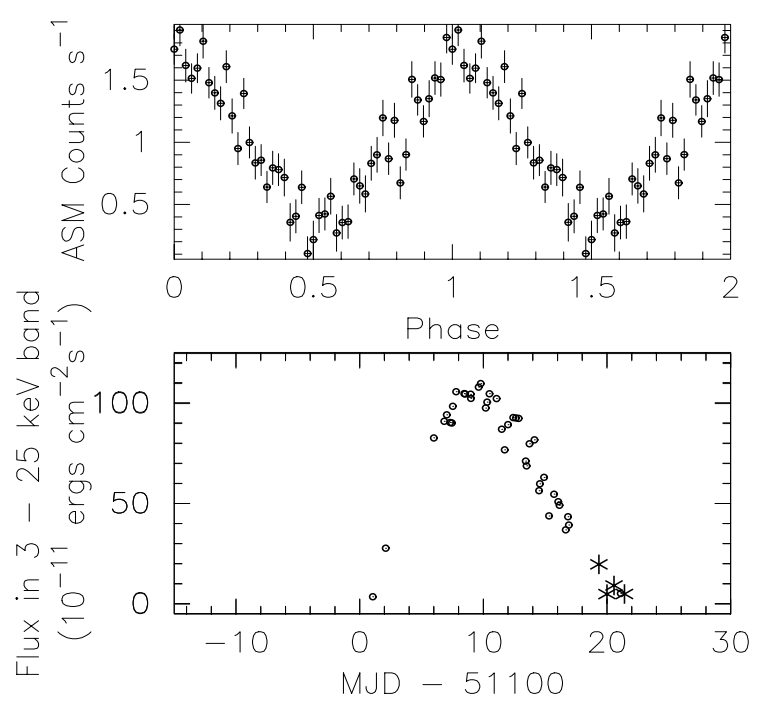

Fig. 1. The folded light curve of LMC X-4 in 1.3-12.1 keV energy band over 30.5 day super-orbital period, obtained from RXTE/ASM data is shown (in upper panel) along with the background-subtracted X-ray flux (averaged over each orbit of the RXTE/PCA observations) in 3-25 keV energy range (bottom panel) during the 1998 OctoberNovember observations. The points marked by "* " are for the observations which were made outside the selected time range and have been included here based on the phase of the super-orbital period. These observations are used to get a better coverage of low intensity state.

of smaller intensity with a duration of $\sim 5$ orbits. From GINGA observations it was found that the iron line intensity is well correlated with the total continuum intensity whereas the iron line equivalent width varies between $0.48 \mathrm{keV}$ to $\simeq 1.37 \mathrm{keV}$ during the 35 day phase (Leahy 2001). As both the X-ray pulsars Her X-1 and LMC X-4 show long periods in similar time scale, we make a comparison of the spectral properties of LMC X-4 with the well known source Her X-1.

In this paper we discuss the spectral variations of LMC X-4 and Her X-1 during the long periods using archival data from the observations with the Proportional Counter Array (PCA) of the RXTE and compare the spectral properties of these two sources.

\section{Observations}

To study the super-orbital phase dependence of various spectral parameters, we have selected RXTE observations of LMC X-4 and Her X-1 at different phases of the super-orbital periods of 30.5 day and 35 day respectively. For LMC X-4, data from RXTE observations made mainly between 1998 October 2 and 1998 November 4 were used for spectral analysis. To have a better coverage of low intensity state (phase) during the super-orbital period, we have also analyzed data from a few other RXTE observations made on 1998 February 6, June 11, August 12, September 9, and December 4. The data used for spectral analysis are out-of-eclipse and free from effects of the flaring state. Figure 1 shows the folded ASM light curve (upper panel) over 30.5 day super-orbital period and the background
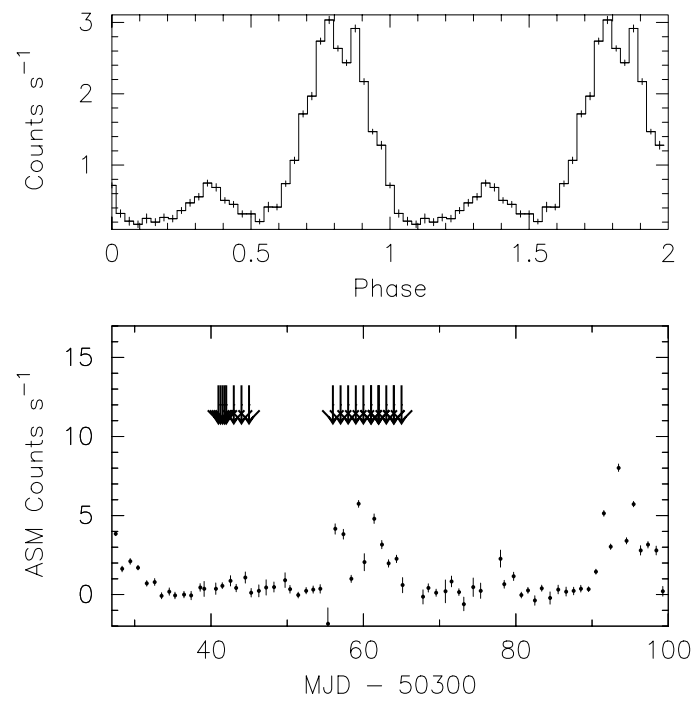

Fig. 2. The folded light curve of Her X-1 in 1.3-12.1 keV energy band over 35 day super-orbital period, obtained from RXTE/ASM data is shown (in upper panel) along with the RXTE/ASM light curve (one day averaged data) of the source for two super-orbital period (bottom panel) which shows the presence of main-on, short-on, and low state. The RXTE/PCA observations used for the spectral analysis are shown by arrows in the figure.

subtracted source flux in 3-25 keV energy band of all the observations used for spectral analysis in the present work. From the figure, it can be seen that the data used for analysis cover a reasonably good part of the 30.5 day super-orbital period. We have shown in Fig. 2 the RXTE/ASM light curve of Her X-1 folded at the 35 day super-orbital period (upper panel) and the RXTE/ASM light curve of the source in the time range of two cycles of the 35 day super-orbital period (bottom panel). A total number of 17 RXTE/PCA pointed observations of Her X-1 covering the main-on, short-on and low states of the superorbital period are used in the present work.

\section{Spectral analysis and results}

\subsection{Data reduction}

Energy spectra in 129 channels were generated from the Standard 2 mode PCA data. The standard procedures for data selection and response matrix generation were followed. Background estimation was done using both bright and faint models of RXTE/PCA according to different intensity states of the source at different phases. We restricted our analysis to the 3-25 keV energy range. Data from all the available, at the time of observation, Proportional Counter Units (PCUs) were added together and response matrices were generated accordingly.

\subsection{Choice of spectral models}

\subsubsection{LMC X-4}

In the energy band of 2-25 keV, the energy spectrum of LMC X-4 is known to consists of a single power law with high 

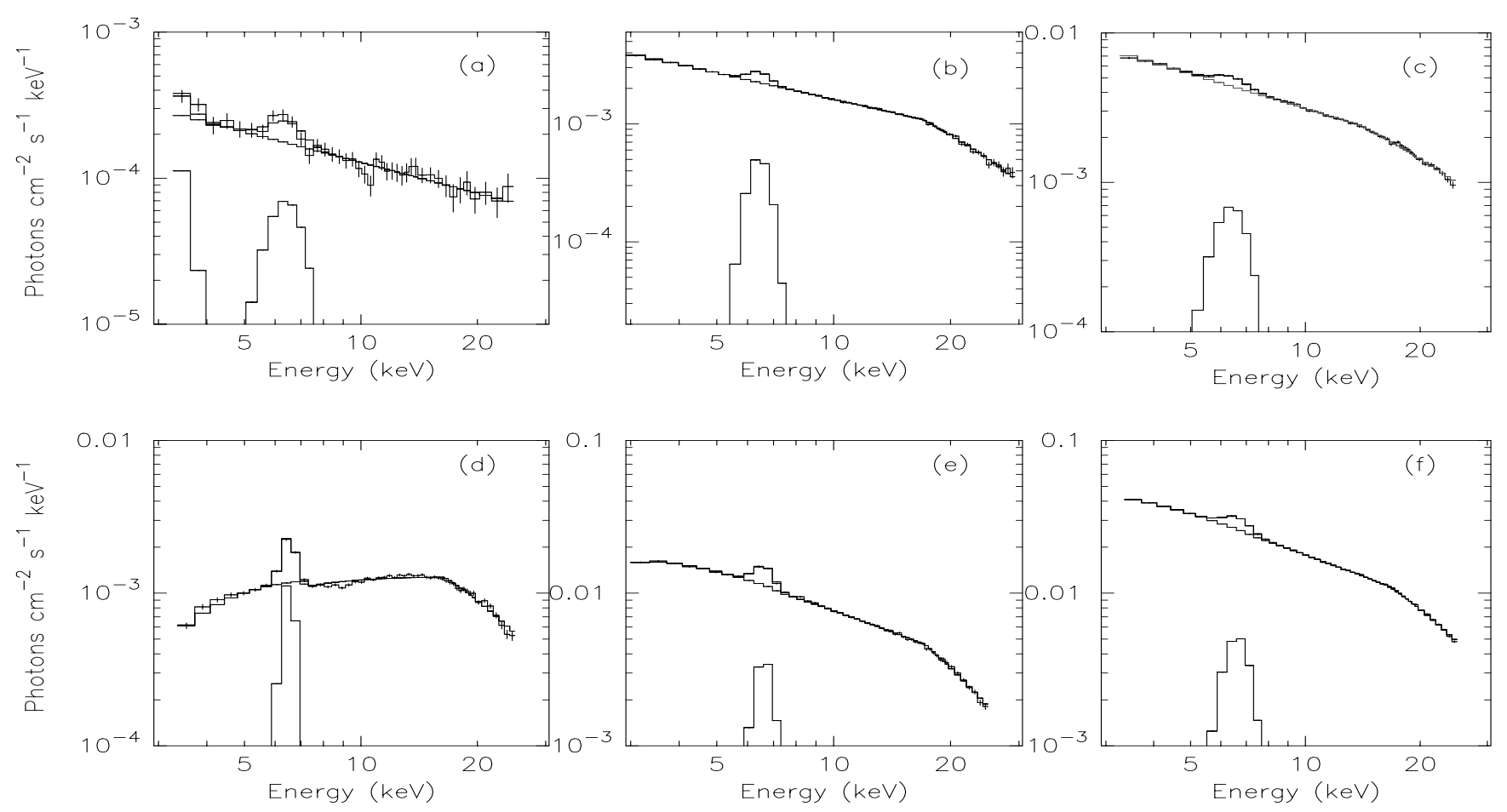

Fig. 3. The deconvolved X-ray spectra of LMC X-4 (top panels) and Her X-1 (bottom panels) during low intensity states a) and d), medium intensity states b) and e) which is known as short-on state for Her X-1 and high intensity states $\mathbf{c}$ ) and f) are shown here. The best fit model consists of a blackbody, a power-law, a Gaussian function for the iron emission line, and a high energy cutoff.

energy cutoff, a soft excess, iron K shell emission line, and low energy absorption. The functional form of the model used for spectral fitting is

$$
\begin{aligned}
I(E)= & \exp \left[-\sigma(E) N_{\mathrm{H}}\right] \\
& \times\left[f_{\mathrm{PF}}(E)+f_{\mathrm{PL}}(E)+f_{\mathrm{FE}}(E)\right] \times f_{\mathrm{hi}}(E)
\end{aligned}
$$

where

$$
\begin{aligned}
f_{\mathrm{PF}}(E) & =\text { Planck function } \\
& =I_{\mathrm{PF}}\left({\frac{E}{E_{\mathrm{PF}}}}^{2}(e-1)\right)\left[\exp \left(\frac{E}{E_{\mathrm{PF}}}\right)-1\right]^{-1}, \\
f_{\mathrm{PL}}(E) & =\text { Power law }=I_{\mathrm{PL}} E^{-\alpha}, \\
f_{\mathrm{FE}}(E) & =\text { Gaussian-shaped iron emission line } \\
& =I_{\mathrm{FE}}\left(2 \pi \sigma_{\mathrm{FE}}^{2}\right)^{-1 / 2} \exp \left[-\frac{\left(E-E_{\mathrm{FE}}\right)^{2}}{2 \sigma_{\mathrm{FE}}^{2}}\right], \\
f_{\mathrm{hi}}(E) & =\text { high energy cut }-\mathrm{off} \\
& =1, \quad \text { if } E<E_{\mathrm{c}} \\
& =\exp \left(-\frac{E-E_{\mathrm{c}}}{E_{\mathrm{f}}}\right), \quad \text { if } E \geq E_{\mathrm{c}} .
\end{aligned}
$$

The value of absorption by the intervening cold material parameterized as equivalent hydrogen column density $N_{\mathrm{H}}$ was kept fixed at $0.055 \times 10^{22} \mathrm{~cm}^{-2}$ which is the Galactic column density towards this source. The blackbody temperature $(k T)$, the center and the width of the iron emission line were fixed at $0.2 \mathrm{keV}, 6.4 \mathrm{keV}$ and $0.65 \mathrm{keV}$ respectively with free normalization (Naik \& Paul 2002).

\subsubsection{Her X-1}

The $2-37 \mathrm{keV}$ energy spectrum of Her X-1 in the main high, short high and low state was measured with GINGA and a two component power-law continuum (absorbed and unabsorbed) model was found to fit the data well (Leahy 2001). However, the best broad-band energy spectrum $(0.1-200 \mathrm{keV})$ measured with the Beppo-SAX does not seem to require the double power-law components and is well described by a model consisting of a single power-law with a high energy cut-off, a blackbody for the low energy excess, two Gaussian iron emission features near $1.0 \mathrm{keV}$ and $6.5 \mathrm{keV}$, and a $\sim 40 \mathrm{keV}$ cyclotron absorption feature (Dal Fiume et al. 1998). Oosterbroek et al. $(2000,2001)$ also used a model, consisting of a soft black-body and a single hard power-law together with two Gaussian emission features near $1.0 \mathrm{keV}$ and $6.5 \mathrm{keV}$, to fit the $0.1-30 \mathrm{keV}$ spectra of Her X-1 from BeppoSAX. We have, therefore, assumed the same spectral model as for LMC X-4. For Her X-1, we have kept the blackbody temperature fixed at $k T=0.1 \mathrm{keV}$ (Vrtilek et al. 1994). However, unlike the case of LMC X-4, the equivalent hydrogen column density, center of the iron emission line, and width of the iron emission line were not fixed.

\subsection{Results}

Figure 3 shows the deconvolved X-ray spectra of LMC X-4 and Her X-1 during low intensity states (Obs. IDs: 30125-04-11-01 and 10055-01-02-00), medium intensity states 
Table 1. Spectral fit parameters of LMC X-4 and Her X-1 during low, medium and high intensity states.

\begin{tabular}{|c|c|c|c|c|c|}
\hline \multirow[t]{2}{*}{ State } & \multirow{2}{*}{$\begin{array}{l}N_{\mathrm{H}}^{1} \\
\left(10^{22} \mathrm{~cm}^{-2}\right)\end{array}$} & \multirow{2}{*}{$\begin{array}{l}\mathrm{Fe}_{\mathrm{c}}^{2} \\
\text { (in } \mathrm{keV} \text { ) }\end{array}$} & \multirow{2}{*}{$\begin{array}{l}W_{0}^{3} \\
\text { (in } \mathrm{keV} \text { ) }\end{array}$} & \multicolumn{2}{|c|}{ Flux $\left(10^{-11} \mathrm{erg} \mathrm{cm}^{-2} \mathrm{~s}^{-1}\right)$} \\
\hline & & & & $7-25 \mathrm{keV}$ & Iron line \\
\hline \multicolumn{6}{|c|}{ Her X-1 } \\
\hline Low & 8.7 & $6.49 \pm 0.03$ & $0.65_{-0.036}^{+0.045}$ & $45.63 \pm 8.26$ & $3.96 \pm 0.75$ \\
\hline Medium & 3.96 & $6.6 \pm 0.04$ & $0.32 \pm 0.03$ & $202.9_{-11.97}^{+15.96}$ & $3.8 \pm 0.3$ \\
\hline High & 1.9 & $6.62 \pm 0.02$ & $0.28 \pm 0.02$ & $530.6 \pm 12.7$ & $7.7 \pm 0.35$ \\
\hline \multicolumn{6}{|c|}{ LMC X-4 } \\
\hline Low & $0.055^{*}$ & $6.4^{*}$ & $0.67_{-0.168}^{+0.082}$ & $4.3_{-0.97}^{+1.15}$ & $0.12 \pm 0.06$ \\
\hline Medium & $0.055^{*}$ & $6.4^{*}$ & $0.322 \pm 0.022$ & $41.7 \pm 1$ & $0.75 \pm 0.09$ \\
\hline High & $0.055^{*}$ & $6.4^{*}$ & $0.26_{-0.02}^{+0.018}$ & $87 \pm 2$ & $1.17 \pm 0.12$ \\
\hline
\end{tabular}

$N_{\mathrm{H}}^{1}$ : Equivalent hydrogen column density, $\mathrm{Fe}_{c}^{2}$ : Iron line energy, $W_{0}^{3}$ : Iron equivalent width.

${ }^{*}$ Parameters are fixed during the spectral fitting as described in the text.
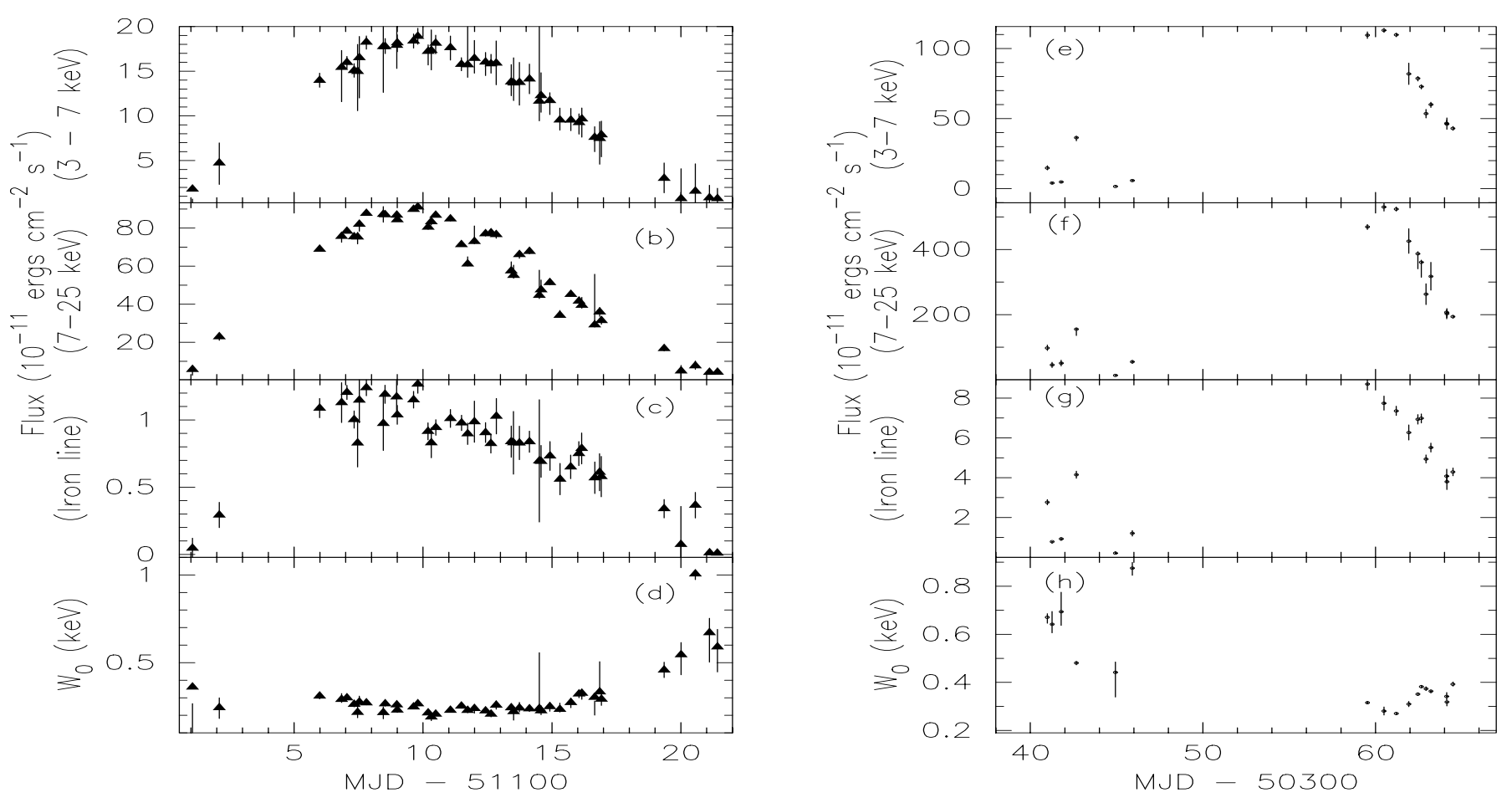

Fig. 4. The figure shows change in the source flux in $3-7 \mathrm{keV}, 7-25 \mathrm{keV}$, iron emission line flux, and iron equivalent width ( $\left.W_{0}\right)$ during the super-orbital period of LMC X-4 (left panels) and Her X-1 (right panels) respectively. The change in the iron emission line flux with the source flux in 7-25 keV energy band are similar for both the sources over the respective super-orbital periods. From the panels (d) and (h), it is seen that the low intensity states are characterized by high value of iron equivalent width whereas the high intensity states are characterized by low value of iron equivalent width which is similar for both the sources.

(Obs. IDs: 30085-01-31-00 and 10055-01-20-00), and high states (Obs. IDs: 30085-01-18-00 and 10055-01-13-00) of the 30.5 day and 35 day super-orbital periods respectively. The presence of prominent iron emission lines in the spectra of two sources during the low intensity states are clearly seen in the panels (a) and (d) respectively. The equivalent width of the iron line is reduced with increase in the intensity for both the sources as seen in the panels (b), (c) and (e), (f) respectively. Although the RXTE/PCA is not sensitive at low energies $(\leq 3 \mathrm{keV})$, the variation in $N_{\mathrm{H}}$ over the 35 day super-orbital period for Her X-1 is very significant (from $1.7 \times 10^{20} \mathrm{~cm}^{-2}$ in high state to $7.3 \times 10^{22} \mathrm{~cm}^{-2}$ in low state). The black-body component is undetected in both sources except in a few low state spectrum of LMC X-4 where the power-law component is comparatively weak.

We have estimated the continuum flux in $7-25 \mathrm{keV}$ and the iron line flux for all the RXTE/PCA observations of LMC X-4 and Her X-1 used in the present work. The center energy, flux, and equivalent width of the iron line, and the continuum flux in 7-25 keV energy band during one representative observation in low, medium, and high intensity states of Her X-1 and LMC X-4 are given in Table 1.

The left panels in Fig. 4 show the continuum flux in 3-7 $\mathrm{keV}$ and 7-25 $\mathrm{keV}$ energy range, the iron emission line 

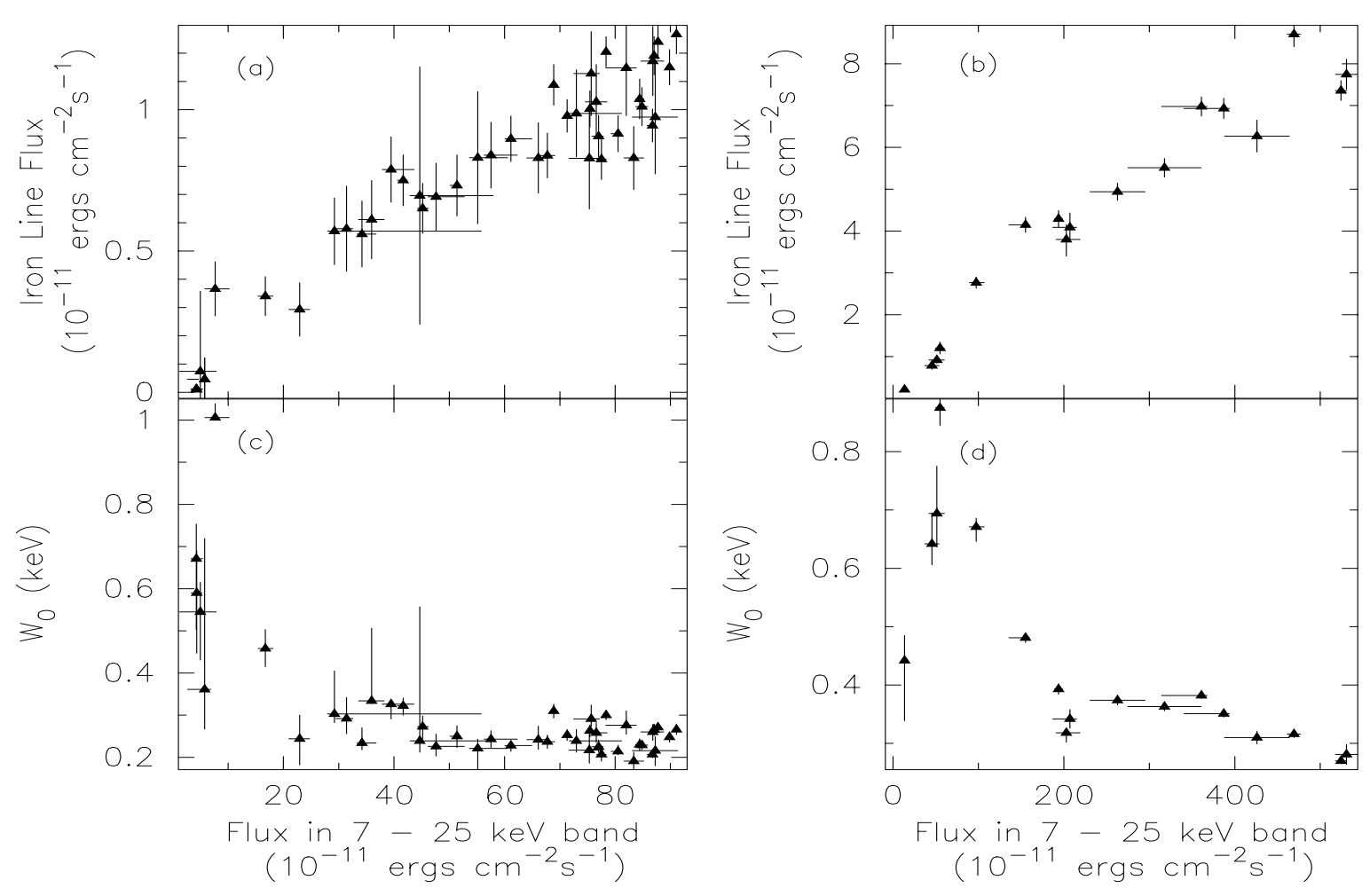

Fig. 5. The figure shows the change in the iron emission line flux and iron equivalent width $\left(W_{0}\right)$ with the source flux in $7-25 \mathrm{keV}$ energy band over the super-orbital period of LMC X-4 (left panels) and Her X-1 (right panels). It is seen that the low intensity states are characterized by high value of iron equivalent width whereas the high intensity states are characterized by low value of iron equivalent width which is similar for both the sources.

flux, and the equivalent width with respect to phases of the super-orbital period of LMC X-4. The same quantities for Her $\mathrm{X}-1$ are shown in the right panels of Fig. 4. From this figure, it can be seen that the relation between hard X-ray flux and iron line flux is remarkably similar in LMC X-4 and Her X-1. In Fig. 5, we show the change in the iron emission line flux and equivalent width as a function of the hard X-ray flux (7-25 keV) in LMC X-4 (left panels) and Her X-1 (right panels) during the 30.5 day and 35 day super-orbital periods respectively. From the upper panels of the Fig. 5, a global proportionality between the line flux and the hard X-ray continuum flux can be seen for both LMC X-4 and Her X-1. Similar type of features have also been observed in Vela X-1 and GX 301-2 (Nagase 1989). Although no clear correlation or anti-correlation is seen between the source flux and the iron line equivalent width $\left(W_{0}\right)$, it is observed that $W_{0}$ of LMC X-4 is very high $(0.4-1.1 \mathrm{keV})$ during low intensity states (source flux $\leq 2.0 \times 10^{-10} \mathrm{ergs} \mathrm{cm}^{-2} \mathrm{~s}^{-1}$ ). During high intensity states, no significant change in $W_{0}$ is observed and its value lies in $0.2-0.4 \mathrm{keV}$ energy range for most of the times of the 30.5 day period. The change in $W_{0}$ with the source flux is similar for Her X-1 during different intensity states of the super-orbital period. It is also observed that the iron line energy is $\geq 6.6 \mathrm{keV}$ during the main-high state of the 35 day super-orbital period of Her X-1 which decreases to $6.4-6.55 \mathrm{keV}$ during the low state of the source. These results are consistent with the previously reported individual measurements of iron line energy from Her X-1. However, no systematic changes are observed in the power-law index, high energy cut-off with the source flux in 7-25 keV for LMC X-4 and Her X-1 during the super-orbital intensity variation. Apart from the change in the hydrogen column density $\left(N_{\mathrm{H}}\right)$ during the super-orbital period of Her X-1 which is constant in LMC X-4 (Lang et al. 1998), the change in all other spectral parameters are similar for both the sources.

\section{Discussion}

Iron emission lines in X-ray pulsars are produced by illumination of neutral or partially ionized material either in (a) accretion disks (mostly seen in LMXB pulsars), (b) stellar wind of the high mass companion star, (c) material in the form of an circumstellar shell, far away from the star, (d) material in the line of sight, or (e) the accretion column. Inoue (1985) and Makishima (1986) estimated the equivalent widths of the fluorescence iron line emission from neutral matter in a sphere surrounding the $\mathrm{X}$-ray source using a power law type incident spectrum. In accretion-powered X-ray pulsars, the iron equivalent width can be higher if the compact object is hidden from direct view by the accretion disk and only $\mathrm{X}$-rays scattered into the line of sight by an accretion disk corona or wind are visible.

If absorbing matter is located between the X-ray source and the observer, the continuum spectrum is absorbed by the matter resulting in increase in the equivalent width monotonically with the column density. This effect was observed in GX 301-2, in 
which a linear correlation between the equivalent width of iron emission line and the column density was found and was interpreted as cold isotropic gas surrounding the compact source (Makino et al. 1985). From ASCA observations of GX 301-2, Endo et al. (2000) found that the iron $\mathrm{K}_{\alpha}$ emission line originates from the region typically at around several Alfvén radii from the neutron star and the observed correlation between the equivalent width of iron $\mathrm{K}_{\alpha}$ line and the line of sight iron column density is consistent with the picture that the fluorescing matter surrounds the pulsar by $\sim 4 \pi$ solid angle. In the HMXB pulsar Vela X-1, the variation in iron line intensity is found to be correlated with the continuum flux variation and the equivalent width of the iron line is found to become relatively large with increase in in-homogeneity of the surrounding matter (Nagase et al. 1986). As Vela X-1 is powered by accretion from stellar wind, three locations other than the accretion disk (as described above) may be responsible for iron line emission. GINGA and ASCA observations of the iron emission line in GX 1+4 suggest the origin to be an isotropically distributed cold matter at a distance of $\sim 10^{12} \mathrm{~cm}$ (Kotani et al. 1999).

In the previous section we presented detection of a positive correlation between the iron emission line flux and the hard X-ray continuum flux in LMC X-4 and Her X-1 and variability of the equivalent width between different intensity states. For Her X-1, this was already reported by Leahy (2001), who inferred that reprocessing of $\mathrm{X}$-rays is important during all intensity levels and the major source of the iron line is the accretion column which is consistent with the accretion-column innerdisk model of Scott et al. (2000). The iron line of Her X-1 is now actually known to consists of two components at $6.41 \mathrm{keV}$ and $6.73 \mathrm{keV}$, which indicates that the observed changes in iron line energies are due to a varying mixture of the two lines (ASCA; Endo et al. 2000). Probably the $6.7 \mathrm{keV}$ line originates in a highly ionized accretion column whereas the $6.4 \mathrm{keV}$ line is associated with the reprocessing in the accretion disk.

The similarities in the variability pattern of iron line flux and hard X-ray continuum flux of Her X-1 and LMC X-4 suggest a similar iron line origin. The identical changes in the iron line parameters over respective super-orbital periods for both the sources reveal the similarities in the distribution of matter in the system causing the iron line emission. However, we note that the hydrogen column density was found to be almost constant over the super-orbital period of LMC X-4 (Lang et al. 1998) which is highly variable in case of Her X-1. This can be explained by the absence of the significant amount of neutral matter along the line of sight. Heindl et al. (1999) explained that in LMC X-4, the flux modulation is caused by the accretion disk and it occurs at the hot and ionized inner disk region where Thomson scattering can remove photons from the beam without affecting the overall spectral shape.

In both the pulsars, the correlation between iron line flux and total flux $(7-25 \mathrm{keV})$ in high state indicates that the iron line is produced near the continuum X-ray source and both are obscured by a precessing accretion disk. However, when the obscuration is very high i.e, in the low state, the equivalent width increases. This indicates that there may be a second, weaker source of iron line away from the hard X-ray source which is not strongly related to the super-orbital intensity variation. Super-orbital phase-resolved observations with good low energy response can help to solve the issue of column density variation.

Acknowledgements. We thank the referee for his useful comments and suggestions which improved the contents of the paper. The work of SN is partially supported by the Kanwal Rekhi Scholarship of the TIFR Endowment Fund. This research has made use of data obtained through the High Energy Astrophysics Science Archive Research Center Online Service, provided by the NASA/Goddard Space Flight Center.

\section{References}

Chevalier, C., \& Ilovaisky, S. A. 1977, A\&A, 59, L9

dal Fiume, D., Orlandini, M., Cusumano, G., et al. 1998, A\&A, 329, L41

Endo, T., Nagase, F., \& Mihara, T. 2000, PASJ, 52, 223

Endo, T. 2000, Ph. D. Thesis

Gerend, D., \& Boynton, P. E. 1976, ApJ, 209, 562

Inoue, H. 1985, Space Sci. Rev., 40, 317

Heindl, W. A., Gruber, D. E., Rothschild, R. E., et al. 1999, BAAS, 31,715

Kelley, R. L., Jernigan, J. G., Levine, A., et al. 1983, ApJ, 264, 568

Kotani, T., Dotani, T., Nagase, F., et al. 1999, ApJ, 510, 369

La Barbera, A., Burderi, L., Salvo, T. Di, et al. 2001, ApJ, 553, 375

Lang, F. L., Levine, A. M., Bautz, M., et al. 1981, ApJ, 246, L21

Lang, F. L., Crannell, C. J., Kaplan, L., et al. 1998, AIP Conf. Proc., 431, 421

Leahy, D. A. 2001, ApJ, 547, 449

Levine, A., Rappaport, S., Putney, A., et al. 1991, ApJ, 381, 101

Li, F., Rappaport, S., \& Epstein, A. 1978, Nature, 271, 37

Makino, F., Leahy, D. A., \& Kawai, N. 1985, Space Sci. Rev., 40, 421

Makishima, K. 1986, in The Physics of Accretion onto Compact Objects, ed. K. O. Mason, M. G. Watson, \& N. E. White, 249

Nagase, F., Hayakawa, S., Sato, N., et al. 1986, PASJ, 38, 547

Nagase, F. 1989, PASJ, 41, 1

Naik, S., \& Paul, B. 2002, JApA, 23, 27

Oosterbroek, T., Parmar, A. N., Orlandini, M., et al. 2001, A\&A, 375, 922

Oosterbroek, T., Parmar, A. N., Dal Fiume, D., et al. 2000, A\&A, 353, 575

Paul, B., \& Kitamoto, S. 2002, JApA, 22, 33

Paul, B., Nagase, F., Endo, T., et al. 2002, ApJ, 597, 411

Pietsch, W., Pakull, M., Voges, W., et al. 1985, Space Sci. Rev., 40, 371

Sanduleak, N., \& Philip, A. G. D. 1977, IAU Circ., 3023

Scott, D. M., Leahy, D. A., \& Wilson, R. B. 2000, ApJ, 539, 392

Vrtilek, S. D., Mihara, T., Primini, F. A., et al. 1994, ApJ, 436, L9

White, N. E. 1978, Nature, 271, 38

Woo, J. W., Clark, G. W., Levine, A. M., et al. 1996, ApJ, 467, 811 